\title{
Research on the spread of awareness of internet rumors based on Bass model
}

\author{
Fengming Liu, Yijie Wang
}

Business School, Shandong Normal University, Jinan, China

Keywords: spread of internet rumors, fluid mechanics, Bass model.

\begin{abstract}
This study proposes to construct the Bass model according to the different stages of the knowledge rate diffusion. Based on the related theories of fluid mechanics, the rumor propagation process is refined and the applicability of the model is improved. Experimental simulation results show that the model can truly reflect the diffusion process of network rumors, and further explain the mechanism of action of each parameter.
\end{abstract}

\section{Introduction}

The spread of Internet rumors is similar to the spread of viruses. En'ko (1889) constructed a model of infectious diseases spread by rumors [1]. In 1927, Kermark and Mokendrick established a standard SIR model [2]. In the 1960s, Daley and Kendall proposed DK model [3]. The "infectiousness" of Internet rumors is enlightening, but the spread of infectious diseases cannot be truly characterized by the principle of infectious diseases, which has led to the correlation and analogy between experts and scholars in different fields. Among them, Mansfield's "infection" theory [4] proposed that technology diffusion is an "infection" process. The doctrine believes that whether companies can adopt technological innovation is largely affected by the behavior of other companies, which reflects the "infectiousness" of technological innovation. In 1969 Frank M. Bass proposed the "infectious" influencing factor of technological innovation, namely the Bass model [5].

In this paper, by analogy with the mechanism of technology diffusion, Internet rumors "awareness rate" is proposed. The "awareness rate" of online rumors refers to the proportional relationship between the group of rumors and the entire group during the spread of online rumors among the crowd. In the early stage of the spread of rumors, a group that had no contact with rumors at first was quickly decomposed into subgroups without use. The focus was on the first reaction of the group and the short-term nature of the time. This paper studies the spreading rules of rumors at different stages, and analyzes the key parameters that affect the spread, in order to provide a theoretical basis for rumor governance decisions.

\section{Bass Network Rumor Propagation Model Based On Fluid Mechanics}

\subsection{Overview of Bass model}

The Bass model divides the population into innovators and imitators. The two types of population are independent and influence each other, and have been affected by the dual factors of mass media and oral communication during the diffusion process. In the early stage of diffusion, mass media was the main influencing factor. Because there were few people who knew about technology in the early stage, and oral communication was dependent on the number of people, obviously it could not dominate the early stage. Innovators have advanced technology adoption due to their adventurous spirit and daring to innovate. This advancement ensures that their decisions are not or less influenced by imitators; in the later stages of diffusion, as the innovator's behavior begins to affect other groups To promote the role, prompt the crowd to actively understand the new technology, and then adopt the new technology, the number of innovation groups continues to increase. At the same time, the number of unknown new technology groups continues to decrease, that is, with the continuous proliferation of new technologies, the number of people adopting new technologies continues to increase, and the effect of oral communication is correspondingly stronger and stronger. Throughout 
the diffusion process, the initial population has independence, while the latter population has mutual influence. The impact of two major factors (mass media and oral communication) has been constantly changing, which is of great reference value for the spread of rumors. Based on the inspiration of the Bass model, the core idea was introduced into the rumor communication research, and the rumor communication was studied in depth.

\subsection{Construction of the Model}

Fluid Mechanics and Information Communication

In physics, as long as a force occurs, there must be a force object and a force object, and the force object is called a source of force. Information dissemination must be carried out under the impetus of a certain source of power, and the dissemination of information will also end at a certain point in the future, which shows that the dissemination of information is hindered. Therefore, the source of power 1 , rumor influence. Rumor's own influence has promoted the spread of rumors in the early days, but this kind of driving force gradually declined with the spread of truth information, that is, the power provided by itself is less and less enough to support the spread of rumors; force source 2, truth influence. The rumor spreads faster than the truth. In 2018, Vosoughi et al. proved that rumors spread much faster than the truth [6]. Force source 3, the demand for group information. The strong demand for information by the crowd has promoted information dissemination. $\mathrm{P}$ is defined as the demand for information of the masses.

The rumor influence is defined as the rumor information density $\rho$, which represents the ratio of the amount of rumor information to the amount of truth information. It is assumed that the rumor information density decays linearly with time as the truth information propagates, that is, $\rho=\rho_{0}-$ $\mathrm{kt} . \rho_{0}$ is the initial information density of rumors, $0<\rho_{0}<1 . \rho_{0}=1$ means that the amount of rumors is the same as the amount of truth. At this time, the rumors have the most influence. $\rho=0$ means that the amount of rumor information is 0 , that is, the rumor has the least influence, and it can be considered that the rumor has disappeared. $\mathrm{k}$ is the decay rate of rumors.

The influence of truth has a certain retarding effect on the spread of rumors, similar to the viscous force in fluid mechanics. Define the truth viscous force $f$. The viscous force $f$ is proportional to the deformation rate, and the viscosity coefficient $\mu$ is introduced, and $f=\mu k$. From Newton's second law, we have:

$$
\frac{d v}{d t}=\frac{P-\mu k}{\rho_{0}-k t}
$$

Simplify further.

$$
\mathrm{v}(\mathrm{t})=\mathrm{v}_{0}+\frac{\mathrm{P}-\mu \mathrm{k}}{\mathrm{k}} * \ln \frac{\rho_{0}}{\rho_{0}-\mathrm{kt}}
$$

Among them, $v$ is the spread rate of rumors, which changes with time, and $v_{0}$ is the initial rate of spread of rumors.

\section{Bass Internet Rumor Propagation Model}

In the model, the people who are exposed to rumors are divided into two categories: positive group and auxiliary group. The former includes people who can rely on their own knowledge to judge the authenticity of information, as well as people who have not yet come into contact with rumors; while the latter is composed of people who mistrust rumors and maliciously create rumors. Pg(t) represents the number of positive groups added at time $t, A g(t)$ represents the number of people added to the rhyme group at time $t, \mathrm{~N}_{\mathrm{Pg}}(\mathrm{t})$ represents the cumulative number of people in the positive group at the cut-off time $t, N_{A g}(t)$ represents the cut-off At time $t$, the helper group accumulates the number of people. Definition $N_{0}$ represents the total number of groups, so $N_{0}-N_{P g}(t)-N_{A g}(t)$ represents the change in personnel after the rumor has been generated. Use $\frac{\mathrm{N}_{\mathrm{Pg}}(\mathrm{t})+\mathrm{N}_{\mathrm{Ag}}(\mathrm{t})}{\mathrm{N}_{0}}$ to represent the rate of change of people who are exposed to rumors, that is, the rate of rumors awareness. At the initial moment, the positive group Pg $(0)=0$ and the assistant group Ag $(0)=0$. 
Define the rumor influence on the group as a stimulus, divided into positive stimulus p and reverse stimulus q. p plays a role in suppressing the spread of rumors. q promoted the spread of rumors. The independent cooling parameter $0<\beta<1$ is defined, which means that some people recognize the rumors information in the process of rumors transmission, and shift from the group camp to the positive group camp.

$$
\begin{aligned}
& \mathrm{p}=\sigma 1 * \frac{\mathrm{v}(\mathrm{t})}{\mathrm{v}_{\mathrm{m}}} \\
& \mathrm{q}=\sigma 2 * \frac{\mathrm{v}(\mathrm{t})}{\mathrm{v}_{\mathrm{m}}}
\end{aligned}
$$

Among them, $0<\sigma 2<1,0<\sigma 1<1$, generally $\sigma 2<\sigma 1$, and $\sigma 1+\sigma 2=1$. $\mathrm{v}_{\mathrm{m}}$ is a selected control speed, which is the maximum speed that all different rumors can propagate. Obviously, for any piece of rumors, $0 \leq \frac{\mathrm{v}(\mathrm{t})}{\mathrm{v}_{\mathrm{m}}} \leq 1$.

The Bass model at time $t$ is:

$$
\begin{aligned}
& \operatorname{Pg}(\mathrm{t})=\left(\mathrm{p}+\mathrm{q} * \beta * \frac{\mathrm{N}_{\mathrm{Pg}}(\mathrm{t})+\mathrm{N}_{\mathrm{Ag}}(\mathrm{t})}{\mathrm{N}_{0}}\right)\left[\mathrm{N}_{0}-\mathrm{N}_{\mathrm{Pg}}(\mathrm{t})-\mathrm{N}_{\mathrm{Ag}}(\mathrm{t})\right] \\
& \mathrm{Ag}(\mathrm{t})=\mathrm{q} *(1-\beta) * \frac{\mathrm{N}_{\mathrm{Pg}}(\mathrm{t})+\mathrm{N}_{\mathrm{Ag}}(\mathrm{t})}{\mathrm{N}_{0}}\left[\mathrm{~N}_{0}-\mathrm{N}_{\mathrm{Pg}}(\mathrm{t})-\mathrm{N}_{\mathrm{Ag}}(\mathrm{t})\right]
\end{aligned}
$$

If the rumor is not diffused among potential people, then $\operatorname{Ag}(\mathrm{t})=0, \beta=1$, (3) can be simplified to the basic form of Bass model:

$$
P g(t)=\left[p+q * \frac{N_{P g}(t)}{N_{0}}\right]\left[N_{0}-N_{P g}(t)\right]
$$

Let $N(t)$ represent the spread of rumors, that is, the sum of the positive group and the rumors group, then the total number of people who are in contact with the rumors at time $t$ is as follows:

$$
N(t)=P g(t)+A g(t)=\left[p+q * \frac{N(t)}{N_{0}}\right]\left[N_{0}-N(t)\right]
$$

Because $\operatorname{Pg}(\mathrm{t}=0)=0$ and $\operatorname{Ag}(\mathrm{t}=0)=0$, that is, $\mathrm{N}(\mathrm{t}=0)=0$, we have:

$$
N(t)=N_{0}\left[\frac{e^{(p+q) t}-1}{e^{(p+q) t}+\frac{q}{p}}\right]
$$

Taking formulas (8)(9) into (3)(4)(7), we can get:

$$
\begin{gathered}
P g(t)=\left[\sigma 1 * \frac{v(t)}{v_{m}}+\sigma 2 * \frac{v(t)}{v_{m}} * \beta * \frac{N_{A g}(t)+N_{P g}(t)}{N_{0}}\right] *\left[N_{0}-N_{P g}(t)-N_{A g}(t)\right] \\
A g(t)=\sigma 2 * \frac{v(t)}{v_{m}} *(1-\beta) * \frac{N_{A g}(t)+N_{P g}(t)}{N_{0}} *\left[N_{0}-N_{P g}(t)-N_{A g}(t)\right] \\
N(t)=N_{0}\left[\frac{e^{(\sigma 1+\sigma 2) \frac{v}{v_{m}} t}-1}{e^{(\sigma 1+\sigma 2) \frac{v}{v_{m}} t}+\frac{\sigma 2}{\sigma 1}}\right]
\end{gathered}
$$

\section{Experimental Simulation And Result Analysis}

\subsection{The overall rationality of the model exists}

Set the initial size of the crowd to 1000.Simulations have shown that the speed of propagation continues to accelerate over time. Taking the time factor as the abscissa, and the proportion of different people in the total population at the corresponding time as the ordinate, obviously when the red curve reaches 1 , it means that the whole social group is exposed to rumors. It can be seen that the contact process of the Internet rumors has an s-shaped curve. This shows that the change of the crowd can be divided into a slow period-a high-speed period-a slow period according to its speed of change, referred to as the SFS stage. 


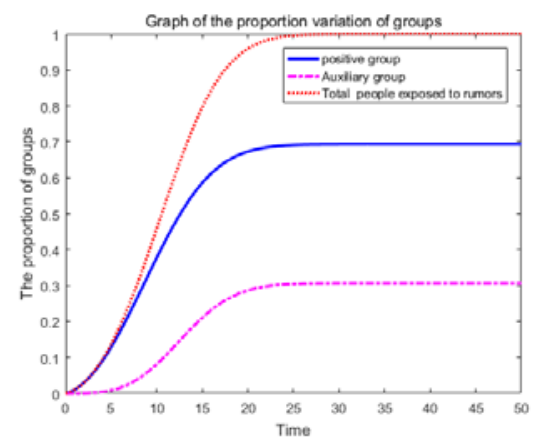

Fig.1 Graph of the proportion variation of groups

By observing the S-shaped curve in Fig. 1, it can be found that if the abscissa does not change and the newly added number of people is taken as the ordinate, then each curve presents a relatively regular bell shape. It can be seen that the process of online rumors contact can be expressed not only by the bell-shaped curve, but also by the S-shaped cumulative curve.

In each early time period, there are only a few rumors contacts, and the S-shaped curve rises slowly; when most members of the system have been in contact, the S-curve accelerates and the increase rate continues to accelerate until it reaches the maximum value ; When more than half of the people in the social system come into contact with online rumors, the S-shaped diffusion curve tends to be flat, because as the proportion of contacts increases, the number of people who are unaware of the online rumors decreases, and fewer and fewer members join the contacts. Come in the ranks. This S-curve is a normal curve because of the effective spread of information, irritating feedback, and the gradual reduction of uncertainty during the spread of rumors.

\subsection{Influence of parameters on the model}

Table 1. The first major parameter setting

\begin{tabular}{|l|l|l|l|}
\hline Situation & 1 & 2 & 3 \\
\hline $\mathbf{P}$ & 2 & 12 & 6 \\
\hline
\end{tabular}

Information demand degree parameter $\mathrm{P}$, truth viscosity coefficient $\mu$, and rumor deformation rate $\mathrm{k}$ are the first major model influence parameters. These three major parameters directly affect the change in the speed of rumor information, which in turn affects the evolution of the overall contact group. However, they will not affect the number and trend of subdivided groups. The space is limited, we list the information demand parameter $\mathrm{P}$ to describe the impact, and the remaining parameters are briefly described.

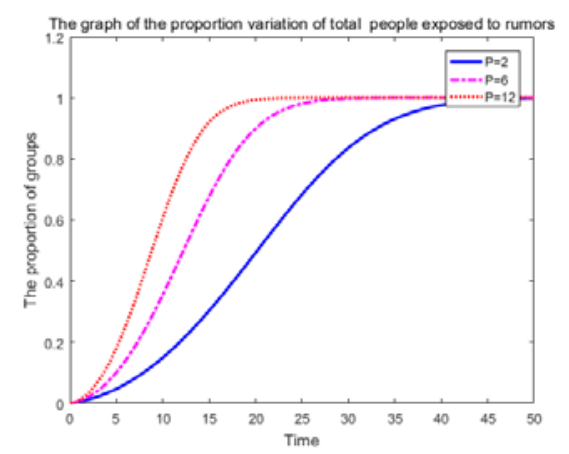

Fig.2 The graph of the proportion variation of total people exposed to rumors

The impact of the demand for P information on the spread of rumors: From the simulation results, we can see that at the same time $t$, as $P$ increases, the speed of rumors spreads accordingly. It can be seen from Fig 2: The crowd starts from the same initial point, and the speed of dividing the proportion of the crowd should increase with the acceleration of P; at the same time, the time for the total contact population to reach steady state is delayed as $\mathrm{P}$ decreases, and the degree of this delay P decreases and 
increases. This shows that people's demand for information can provide power for the spread of rumors, thereby speeding up the spread. The degree of information demand shows that the more relevant the public's daily life or work area is, the more interested the public is and the higher the degree of demand for it.

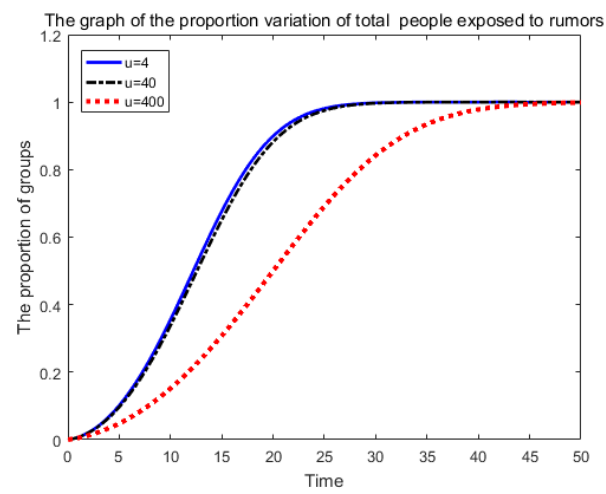

Fig.3 The graph of the proportion variation of total people exposed to rumors

The influence of $\mu$ on the spread of rumors: From Fig 3, the more similar the rumors are to the truth, the larger the value of $\mu$. The increase of $\mu$ means that the drag resistance of the true relative rumors increases, so the spread rate of the rumors is smaller. The curve representing higher $\mu$ values in Fig 3 takes a significantly slower time to reach steady state, while the curves represented by other smaller values almost coincide. This shows that if one of the two rumors is very detailed and the other is very vague, there will be a significant difference in the speed of propagation of the two, and the change in the total population will also be significantly different, but if the two rumors are similar or different Not enough, then this difference has minimal impact on the spread of rumors.

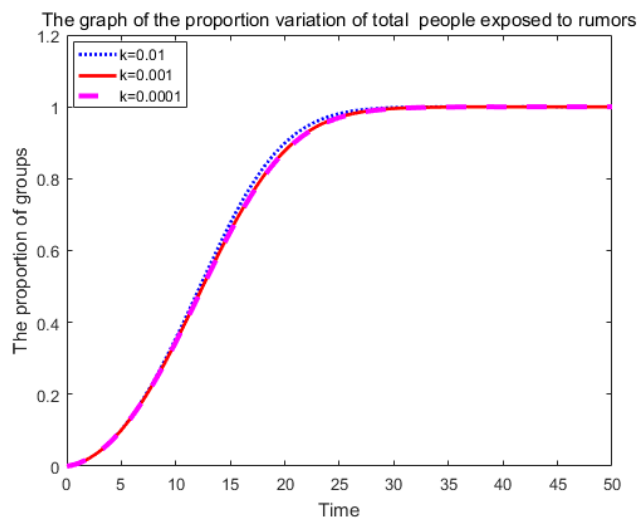

Fig. 4 The graph of the proportion variation of total people exposed to rumors

The effect of $k$ on the spread of rumors: Simulation shows that the difference in the speed of rumors is mainly due to the nature of rumors. When rumors enter the crowd, human subjective factors account for most of the impact. Wisdom should be based on understanding. However, due to subjective limitations, people's understanding of rumors may not be correct and in place. According to the almost coincident curve in Figure 4, the influence of individual ideas is much stronger than the influence of the nature of the rumors, so the rumors information is not sensitive to changes in the crowd. reasonable. And the propagation speed simulation also shows that the model is not very sensitive to k-value movement.

The cooling parameter $\beta$ and the stimulation parameters $p$ and $q$ are the second type of influence parameters. It can be seen from the graph of the first type of parameters that the curves are completely coincident, that is, changes in the stimulation coefficient and irrational judgment of cooling parameters will not affect the speed and trend of the total contact group changes, because these two coefficients are only positive groups and help The increase and decrease of the rumor groups will not affect the overall contact groups. 
The influence of $\mathrm{p}$ and $\mathrm{q}$ on the spread of rumors: The simulation shows that the number of subdivided groups is affected by both stimulus coefficients. The increase of $\mathrm{p}$ will lead to a positive growth, and the increase of q coefficient will lead to the growth of the group of rumors. It can be further observed that the changes of the subdivided population are very sensitive to $\mathrm{p}$ and $\mathrm{q}$, and the stimulation parameters should obviously be classified as key influencing factors. When the spread of rumor information occurs, we should strive to increase $\mathrm{p}$ and control q so that the rumor information will not rise to a large probability that affects the thinking of most people in the entire group.

The effect of cooling parameter $\beta$ on the speed of population change: when the forward stimulation parameter and the reverse stimulation parameter are the same, the two groups do not account for $50 \%$ each, because $\beta$ makes some of the rhyme groups restore their reason to the positive group. With the increase of $\beta$, the rumors group shrinks and the positive group grows. The difference in the value of $\beta$ is up to about 10 times, the difference is not significant; the simulation shows that as the value of $\beta$ increases, the incidence of unreasonable first impressions is lower, so the segmented population is more sensitive to changes in $\beta$; As the $\beta$ value increases, the model becomes more sensitive to it.

In the initial stage, positive crowd growth preceded the rumors, because the majority of citizens have a correct understanding of information. Although there are obvious differences in the number of the same group, all the positive groups and all the rhyme groups enter the steady state at the same time. The cooling parameters affect the distribution of the people in the steady state. Obviously, the cooling parameters should be increased. To sum up, it is necessary to improve the popularization and education of science popularization and bring the overall cultural level of society to a new level.

The parameter sensitivity table (Table II) finds the entry point for rumor control at this stage, which has important reference value for the subsequent research on the development trend of rumor.

Table 2. Parameter Sensitivity Table

\begin{tabular}{|c|c|c|c|c|c|c|}
\hline Parameters & $\mathbf{P}$ & $\boldsymbol{\mu}$ & $\mathbf{k}$ & $\boldsymbol{\beta}$ & $\mathbf{p}$ & $\mathbf{q}$ \\
\hline sensitivity & $\sqrt{ }$ & & & & $\sqrt{ }$ & $\sqrt{ }$ \\
\hline Monsitive & & & & $\sqrt{ }$ & & \\
\hline Not too sensitive & & $\sqrt{ }$ & $\sqrt{ }$ & & & \\
\hline
\end{tabular}

\section{Conclusion}

This paper builds a rumor diffusion model based on the BASS model, and simulates and analyzes the rationality of the model and the influence of parameters. The research results of this paper are not only a detailed and thorough analysis of the various mechanisms of the various parameters in the diffusion rate of the awareness rate, such as the steady-state arrival time and the change of the subdivided population, but also to further distinguish and summarize the model for different The sensitivity of the parameter. A comprehensive and in-depth analysis of the model has been made to provide a theoretical basis for related research.

\section{References}

[1] Jin Zhen, Pan Jinxiao. Research on epidemic model with continuous vaccination [J]. Journal of Applied Basic and Engineering Sciences, 2001(Z1): 120-124

[2] Keimack W O,McKendrick A G. Contributions to the mathematical theory of epidemics,part I[J].Proceedings of the Royal Sosiety of London A,1927,115:700-721.

[3] Pittel B. On a Daley-Kendall model of random rumours[J]. Journal of Applied Probability,1990, 27(1):14-27.

[4] Mansfield. E. Technology change and the rate of imitation[J].Econometrics,1961(29):741-765. 
[5] Bass,F. M. (1969). A new product growth for model consumer durables. Management Science, 15(1):215-227.

[6] Soroush Vosoughi, Deb Roy,Sinan Aral. The spread of true and false news online. Science,2018 DOI: 10.1126/science.aap9559 